\title{
Precut fistulotomy for choledocholithiasis: what about long-term efficacy?
}

\section{다 (i)}

\author{
Authors \\ Paraskevas Gkolfakis, Marianna Arvanitakis
}

Institution

Department of Gastroenterology Hepatopancreatology and

Digestive Oncology, Erasme University Hospital, Université

Libre de Bruxelles, Brussels, Belgium

submitted 1.6.2019

accepted after revision 5.6.2019

Bibliography

DOI https://doi.org/10.1055/a-0959-6105 |

Endoscopy International Open 2020; 08: E231-E233

\section{(c) Georg Thieme Verlag KG Stuttgart · New York elSSN 2196-9736}

\author{
Corresponding author \\ Marianna Arvanitakis, MD PhD, Department of \\ Gastroenterology, Hepatopancreatology and Digestive \\ Oncology, Erasme University Hospital, Université Libre de \\ Bruxelles, Route de Lennik 808, 1070 Brussels, Belgium \\ Fax: +3225554697 \\ Marianna.Arvanitaki@erasme.ulb.ac.be
}

Patients with choledocholithiasis present with a variety of symptoms ranging from biliary colic to severe acute pancreatitis and cholangitis. Endoscopic retrograde cholangiopancreatography (ERCP) is considered the gold standard to treat them [1]. Simple ERCP cannulas or bendable papillotomes have been developed to facilitate access to the common bile duct (CBD), followed by incision of the sphincter allowing stone extraction using balloon extractor catheters or basket wires.

In approximately $20 \%$ of patients undergoing ERCP, CBD cannulation may fail due to difficult cannulation [2], defined as more than five cannulation attempts, or attempts lasting more than 5 minutes, or two or more unintentional pancreatic guidewire passages. If the pancreatic duct is repeatedly cannulated or injected, the pancreatic guidewire (PGW)-assisted technique is recommended [3]. However, the pancreatic duct is not always easily accessible, and in those cases, experienced endoscopists resort to one of the precutting techniques (precut fistulotomy and conventional precut technique) based on a patient's characteristics (e.g. papilla's size and morphology) and endoscopist expertise [4, 5].

Precut fistulotomy (PF) aims to avoid injuring the distal part of the sphincter (including the pancreatic duct) by using a needle-knife to perform a stepwise incision of the mucosa. The incision starts approximately $3 \mathrm{~mm}$ over the roof of the papilla, followed by an upward or downward cut until the biliary sphincter or the bile duct is visualized. On the other hand, the conventional precut technique consists of a stepwise incision of the mucosa starting at the upper end of the orifice in the expected direction of the bile duct until visualization of the whitish onion skin-appearing sphincter muscle. Both techniques increase the rate of success with selective biliary cannulation, but they have also been identified as risk factors for post-ERCP pancreatitis (PEP) [6].

Choledocholithiasis recurrence after ERCP is not rare [7]. Risk factors include, among others, the CBD diameter; presence of biliary stricture, gallstones or peri-ampullary diverticulum; and sphincterotomy itself [8]. Whether precut fistulotomy, by retaining integrity of the distal papillary sphincter, could also act as a potential risk factor for CBD stones recurrence remains unknown. This question is being elucidated in the current issue of Endoscopy International Open by Archibugi et al. [9].

In their retrospective study the authors included 85 patients with a diagnosis of choledocholithiasis treated by CBD clearance after PF. These cases were age and sex matched with respective patients fulfilling inclusion criteria but undergoing conventional endoscopic biliary sphincterotomy (BS). Primary and secondary endpoints consisted of stone recurrence rate (defined as the need to repeat ERCP or new admission for biliary event) and complication rate, respectively. Two experienced endoscopists performed all examinations.

No difference was shown in long-term disease recurrence (14.1\% vs. $12.9 \%$ for PF and BS groups, respectively) and ERCP repetition probability between the two groups. However, mean time to recurrence was significantly shorter in the PF group (approximately 2.5 months compared to 2 years in the BS group)! As already mentioned, different risk factors for post-ERCP CBD stone recurrence have been identified. The difference shown by the authors in the time of recurrence between the groups could be explained by the fact that the narrower intact distal sphincter after PF may hinder spontaneous clearance of fragments during the physical history of the disease. It is noteworthy that 
the majority of patients underwent cholecystectomy before or after ERCP, and that there was no significant difference between the two groups $(98.1 \%$ vs. $87.7 \%[P=0.06]$ for the PF and BS groups, respectively). Another hypothesis could be that earlier recurrence rate occurs not due to new stones that fail to go through the intact sphincter but due to residual accumulating fragments. Indeed, the only factor acting protectively against ERCP repetition for stone recurrence was the confirmed CBD clearance. The authors used occlusion cholangiogram to assess CBD clearance. In patients undergoing PF, the combination of flow alteration together with contractility of the distal part of the sphincter may obscure residual fragments or small stones during occlusion cholangiogram. Whether it is the ideal method to evaluate CBD clearance in this subgroup of patients has not been proven. Irrigation of the bile duct with normal saline at the end of the procedure could be helpful to reduce the rate of residual CBD stones without adding significant burden to the procedure, as shown in both a prospective and a retrospective study $[10,11]$. In addition, wider extension of cut site after fistulotomy, if feasible, should also be applied to facilitate passage of residual fragments. Finally, post-procedural imaging and laboratory follow-up could be proposed to confirm definitive stone clearance, and advanced techniques such as peroral cholangioscopy could be used [12].

Applying precut techniques demands a high level of expertise and even when performed by a skilled and experienced endoscopist, it has been associated with an increased risk of PEP. This has been mainly attributed to the fact that precut was applied after a number of previous cannulation attempts that caused trauma and edema at the level of the pancreatic orifice. In fact, "moving" the precut technique earlier during a difficult cannulation has been associated with a decreased risk of PEP [13]. Moreover, PF compared to conventional precut technique significantly decreased the PEP rate in a systematic metaanalysis [14]. The most reasonable explanation is that during conventional precut, the pancreatic orifice is not protected and injuries may occur. In the current study, the authors applied a mix of early and delayed precut based on operator's choice and protocols running during the study. A slightly increased number of perforations and cases of post-ERCP pancreatitis were reported in the PF group compared to the BS group ( $3.6 \%$ vs. $1.2 \%$ and $12.9 \%$ vs. $7.1 \%$, respectively), but these differences were not statistically significant. It is worth noting that the only factor independently associated with PEP was cannulation of the main pancreatic duct. Of interest, in the current study, the main pancreatic duct was cannulated in 49 of 170 patients (28.8\%) but a prophylactic pancreatic stent was placed in only 28 of them. On the contrary, rectal indomethacin was administered in all patients with no contraindications during almost the whole study period.

Finally, in the study's referral center, precut fistulotomy was applied in $12.8 \%$ of the ERCPs over a decade. However, among them, 49 patients ( 31 vs. 18 for the PF and BS groups, respectively) experienced at least one unintentional pancreatic duct cannulation. Based on current recommendations, we may speculate that using the PGW-assisted technique for these patients before proceeding to precut could further decrease both the need to perform a precut fistulotomy and the PEP rate, especially if combined with prophylactic pancreatic stent placement whenever indicated.

\section{Acknowledgements}

Dr. Gkolfakis acknowledges the Hellenic Society of Gastroenterology for providing an educational support grant on therapeutic endoscopy.

\section{Competing interests}

None

References

[1] Chathadi KV, Chandrasekhara V. Committee ASoP. et al. The role of ERCP in benign diseases of the biliary tract. Gastrointest Endosc 2015; 81: $795-803$

[2] Tse F, Yuan Y, Moayyedi P et al. Guidewire-assisted cannulation of the common bile duct for the prevention of post-endoscopic retrograde cholangiopancreatography (ERCP) pancreatitis. Cochrane Database Syst Rev 2012; 12: CD009662

[3] Testoni PA, Mariani A, Aabakken L et al. Papillary cannulation and sphincterotomy techniques at ERCP: European Society of Gastrointestinal Endoscopy (ESGE) Clinical Guideline. Endoscopy 2016; 48: $657-683$

[4] Berry R, Han JY, Tabibian JH. Difficult biliary cannulation: Historical perspective, practical updates, and guide for the endoscopist. World J Gastrointest Endosc 2019; 11: 5-21

[5] Verma D, Gostout C], Petersen BT et al. Establishing a true assessment of endoscopic competence in ERCP during training and beyond: a single-operator learning curve for deep biliary cannulation in patients with native papillary anatomy. Gastrointest Endosc 2007; 65: 394 400

[6] Rustagi T, Jamidar PA. Endoscopic retrograde cholangiopancreatography (ERCP)-related adverse events: post-ERCP pancreatitis. Gastrointest Endosc Clin N Am 2015; 25: 107 - 121

[7] Konstantakis C, Triantos C, Theopistos V et al. Recurrence of choledocholithiasis following endoscopic bile duct clearance: Long term results and factors associated with recurrent bile duct stones. World ] Gastrointest Endosc 2017; 9: 26-33

[8] Cai JS, Qiang S, Bao-Bing Y. Advances of recurrent risk factors and management of choledocholithiasis. Scand J Gastroenterol 2017; 52: $34-43$

[9] Archibugi L, Mariani A, Capurso G et al. Needle-knife fistulotomy vs. standard biliary sphincterotomy for choledocholithiasis: bile duct stone recurrence and complication rate. Endosc Int Open 2019; 07: E1733-E1741

[10] Ahn DW, Lee SH, Paik WH et al. Effects of saline irrigation of the bile duct to reduce the rate of residual common bile duct stones: a multicenter, prospective, randomized study. Am J Gastroenterol 2018; 113: $548-555$

[11] Jang SE, Ahn DW, Lee SH et al. Preventive saline irrigation of the bile duct after the endoscopic removal of common bile duct stones. Dig Dis Sci 2013; 58: 2353-2360

[12] Anderloni A, Auriemma F, Fugazza A et al. Direct peroral cholangioscopy in the management of difficult biliary stones: a new tool to confirm common bile duct clearance. Results of a preliminary study. J Gastrointestin Liver Dis 2019; 28: 89-94 
[13] Lopes L, Dinis-Ribeiro M, Rolanda C. Early precut fistulotomy for biliary access: time to change the paradigm of "the later, the better"? Gastrointest Endosc 2014; 80: 634-641
[14] Choudhary A, Winn J, Siddique S et al. Effect of precut sphincterotomy on post-endoscopic retrograde cholangiopancreatography pancreatitis: a systematic review and meta-analysis. World J Gastroenterol 2014; 20: $4093-4101$ 